\title{
Relation between gastric emptying rate and rate of intraluminal lipolysis
}

\author{
B D Maes, Y F Ghoos, B J Geypens, M I Hiele, P J Rutgeerts
}

\begin{abstract}
The variable gastric emptying rate of a test meal is one of the major problems in evaluating accurately gastrointestinal physiological functions beyond the stomach. The aim of this study was to evaluate the effect of the gastric emptying rate on the rate of intraluminal lipolysis. Thirty four subjects without pancreatic disease (21 with a normal gastric emptying and 13 with a known slow gastric emptying) and 14 subjects with pancreatic disease (four without and 10 with pancreatic insufficiency) were studied using a dual labelled breath test. The test meal consisted of one egg, 60 grams of white bread, 10 grams of margarine, and $150 \mathrm{ml}$ of water $(350 \mathrm{kcal})$. The egg yolk was labelled with $91 \mathrm{mg}$ of ${ }^{13} \mathrm{C}$-octanoic acid, the margarine was labelled with $296 \mathrm{kBq}$ of distearyl-2-14 Coctanoyl-glycerol. Breath samples were taken every 15 minutes during six hours and analysed for ${ }^{13} \mathrm{CO}_{2}$ and ${ }^{14} \mathrm{CO}_{2}$ content. The gastric emptying rate of the meal was evaluated by the gastric emptying coefficient, the half emptying time, and the lag phase; the rate of intraluminal lipolysis was evaluated by the six hours cumulative ${ }^{14} \mathrm{CO}_{2}$ excretion. Despite a clear distinction in the rate of intraluminal lipolysis, no difference could be detected in gastric emptying rate of the test meal between subjects without and with pancreatic disease. In subjects with pancreatic insufficiency, intraluminal hydrolysis was the rate limiting process in fat assimilation; in patients without pancreatic insufficiency, however, gastric emptying could be rate limiting. Therefore, patients with known slow gastric emptying, displayed a significantly decreased rate of intraluminal lipolysis compared with normal controls. This decrease could be corrected for accurately using a correction factor based on the gastric emptying coefficient. In conclusion, the combined ${ }^{13} \mathrm{C}$-octanoic acid and ${ }^{14} \mathrm{C}$-mixed triglyceride breath test permits the measurement of gastric emptying rate and intraluminal lipolysis simultaneously in a minimally invasive way. Correction of intraluminal lipolysis rate for gastric emptying rate of the given test meal permits evaluation of fat assimilation rates in a physiological way regardless of gastric emptying disorders. (Gut 1996; 38: 23-27)
\end{abstract}

Keywords: gastric emptying, intraluminal lipolysis, breath test.
Fats make up a major part of the total energy intake. ${ }^{1}$ The assimilation of fat is a complex process, because of the hydrophobic nature of fat constituents. Nevertheless, dietary fat absorption is usually very efficient. Some fat hydrolysis takes place in the stomach $(10-30 \%)$ by the action of lingual and gastric lipase, ${ }^{2-5}$ but fat is digested and absorbed mainly in the proximal small intestine through the action of pancreatic lipase-colipase, and carboxyl ester lipase. ${ }^{6}$ With increased load, however, fat is also absorbed in the distal small intestine. ${ }^{7}$ After hydrolysis, the fatty acids and 2-monoglycerides are easily dispersed by bile salts into micelles, followed by a rapid diffusion through the brush border membrane. ${ }^{8}{ }^{9} \mathrm{In}$ the absence of bile salts, dietary fat absorption is reduced by $30-40 \%$; if pancreatic lipases are also absent, fat absorption is reduced by $70 \% .{ }^{1011}$

Before digestion, fat needs to be emulsified. ${ }^{12}$ Emulsification of fat in droplets occurs in the stomach by mechanical shearing. Smaller droplets are stabilised by fatty acids (produced during gastric lipolysis), by proteins, and other amphipathic components in the stomach content. ${ }^{13} 14$ The fatty acids produced in the stomach also accelerate binding of pancreatic lipase-colipase to fat droplets and induce the release of cholecystokinin, inducing gall bladder contraction, relaxation of the sphincter of Oddi, and pancreatic enzyme secretion. ${ }^{15}$ As part of an ordinary mixed meal, water and fat have different patterns of emptying, water leaving the stomach first. When emulsified, fat leaves the stomach together with the liquid phase. ${ }^{16} 17$ Extracellular fat empties predominantly as an oil phase at a rate similar to that of an equicaloric digestible solid meal. 1718

One important variable influencing the rate of fat assimilation is gastric emptying of the ingested fat load. The aim of this study was to evaluate the impact of the gastric emptying rate on the rate of intraluminal lipolysis in normal subjects, patients with known slow gastric emptying, and subjects with pancreatic disease; and hence, we aimed at evaluating intraluminal lipolysis regardless of gastric emptying rates.

\section{Methods}

\section{Subjects}

Thirty four subjects without pancreatic disease and 14 patients with confirmed pancreatic disease underwent a dual labelled breath test. Twenty one normal healthy volunteers (11 women, 10 men, aged 18 to 45 years) were 
studied as well as 13 patients with known slow gastric emptying rate of solids (nine women, four men, aged 20 to 51 years); the diagnosis of delayed gastric emptying had been made by radioscintigraphy and by the ${ }^{13} \mathrm{C}$-octanoic acid breath test. ${ }^{19}$ Three patients had a gastric outlet syndrome (peptic ulcer disease), while the other 10 subjects suffered from an idiopathic gastroparesis.

Fourteen patients with chronic pancreatitis were studied (three women, 11 men, aged 35 to 56 years). Pancreatic disease was caused by alcohol in 12 patients and in two the cause was not identified. The diagnosis of chronic pancreatitis was based on the presence of pancreatic calcifications (computed tomography), typical ERCP findings or the diagnosis was made at surgery. Three patients had undergone pancreatic surgery. Four patients had normal fat excretion in stools and 10 had a faecal fat excretion $>7 \mathrm{~g} /$ day. ${ }^{20}$ Two patients with pancreatic disease also had endocrine insufficiency with insulin dependent diabetes mellitus.

None of the volunteers were taking any drugs or had a medical or surgical history. Three patients with delayed gastric emptying were taking omeprazole 20 to $40 \mathrm{mg}$ a day. Nine patients with pancreatic disease were treated with pancreatic enzyme supplements; two patients had insulin substitution treatment; one patient was taking narcotic analgesics. Apart from the insulin substitution treatment, no medication was permitted during the 24 hours preceding the test. Informed consent was obtained from all subjects. The study protocol was approved by the ethical committee of the University of Leuven.

\section{Test meal}

After an overnight fast of 12 hours, each subject was studied by a dual carbon labelled breath test to measure the gastric emptying rate of solids and the intraluminal lipase activity simultaneously. The test meal consisted of one egg, 60 grams of white bread, and 10 grams of margarine. Some $91 \mathrm{mg}$ of ${ }^{13} \mathrm{C}$ octanoic acid (Isotec, Ohio, USA) was added to and homogenised in the egg yolk and baked separately from the egg white, to ensure a firm retention of the label in the solid phase of the meal. ${ }^{19}$ Some $296 \mathrm{kBq}$ of the mixed triglyceride 1,3-distearyl, $2\left[{ }^{14} \mathrm{C}\right]$ octanoylglycerol ( $\left.{ }^{14} \mathrm{C}-S O S\right)$ ( $\mathrm{K} \mathrm{U}$ Leuven, department of chemistry, Leuven, Belgium) was added to and homogenised in the margarine. The total energy content of the meal was $350 \mathrm{kcal}$. The meal was consumed within 10 minutes, immediately followed by drinking $150 \mathrm{ml}$ of water. The subjects were not allowed to eat or drink during the following six hours.

\section{Study design}

Breath samples were taken before the meal and at 15 minute intervals for a six hour period after ingestion of the test meal. At each sampling time, two samples were taken. The first sample, for detection of ${ }^{13} \mathrm{CO}_{2}$, was collected in an aluminium coated air bag of three litres (Tesseraux, Bürstadt, Germany). The second sample, for the detection of ${ }^{14} \mathrm{CO}_{2}$, was collected by blowing air through a pipette in a plastic vial containing two $\mathrm{mmol}$ of hyamine hydroxide, and thymolphtaleine as indicator; blowing lasted until decoloration of the indicator, corresponding with the captation of two $\mathrm{mmol}$ of $\mathrm{CO}_{2}$. The ${ }^{13} \mathrm{C}$ enrichment was determined by isotope ratio mass spectrometry (model 250, Finnigan MAT, Bremen, Germany). The $\delta$ values were expressed versus the PDB-international standard, after correction for oxygen isotopes, and were calculated as percentage of recovery per hour of the administered dose, assuming the $\mathrm{CO}_{2}$ production to be $300 \mathrm{mmol} / \mathrm{m}^{2}$ of body surface area per hour. Body surface area was calculated using the weight-height formula of Haycock et $a l .{ }^{21}$ The ${ }^{13} \mathrm{CO}_{2}$ excretion data were further analysed by non-linear regression analysis for curve fitting and calculation of different parameters describing the gastric emptying rate of solids - that is, the gastric emptying coefficient (GEC), the half emptying time $\left(t_{1 / 2}\right)$, and the lag phase (tlag). Details were published elsewhere. ${ }^{19}$

The ${ }^{14} \mathrm{C}$ activity was measured by $\beta$-scintillation counting (model 2450, Packard, Packard Instrument Company, Downers Grove, Illinois, USA) after adding $10 \mathrm{ml}$ of Hionic fluor (Packard Instrument Company, Downers Grove, IL, USA) to the vials and with correction for quenching; the results were expressed as percentage of ${ }^{14} \mathrm{CO}_{2}$ activity per hour of the administered dose and as cumulative values over six hours. ${ }^{22}$ Correction of the cumulative dose ${ }^{14} \mathrm{CO}_{2}$ for the gastric emptying rate was made using the following empirical formula:

corrected cumulative dose $=$
cumulative dose $\times\left(1+\frac{\left(\mathrm{GEC}_{\text {normal }}-\mathrm{GEC}_{\text {subject }}\right)}{\mathrm{GEC}_{\text {normal }}}\right)$,

with $\mathrm{GEC}_{\text {normal }}$, the mean gastric emptying coefficient of the control group and $\mathrm{GEC}_{\text {subject }}$, the gastric emptying coefficient of the subject examined.

\section{Statistical analysis}

The Mann-Whitney-Wilcoxon and the signed rank tests (SAS: PROC NPAR1WAY and PROC UNIVARIATE ${ }^{23}$ ) were used to detect differences in ${ }^{13 / 14} \mathrm{CO}_{2}$ excretion data, the gastric emptying parameters, and cumulative dose of ${ }^{14} \mathrm{CO}_{2}$ excreted over six hours (as a parameter of intraluminal lipolysis) between the different groups of subjects.

\section{Results}

Figure 1 shows the mean ${ }^{13} \mathrm{CO}_{2}$ (originating from ${ }^{13} \mathrm{C}$-octanoic acid) and ${ }^{14} \mathrm{CO}_{2}$ (originating from ${ }^{14} \mathrm{C}-\mathrm{SOS}$ ) excretion curves of the subjects without and the subjects with pancreatic disease. In both groups, there was no difference in ${ }^{13} \mathrm{C}$ excretion (Fig $1 \mathrm{~A}$ ) between subjects with or without pancreatic disease at 

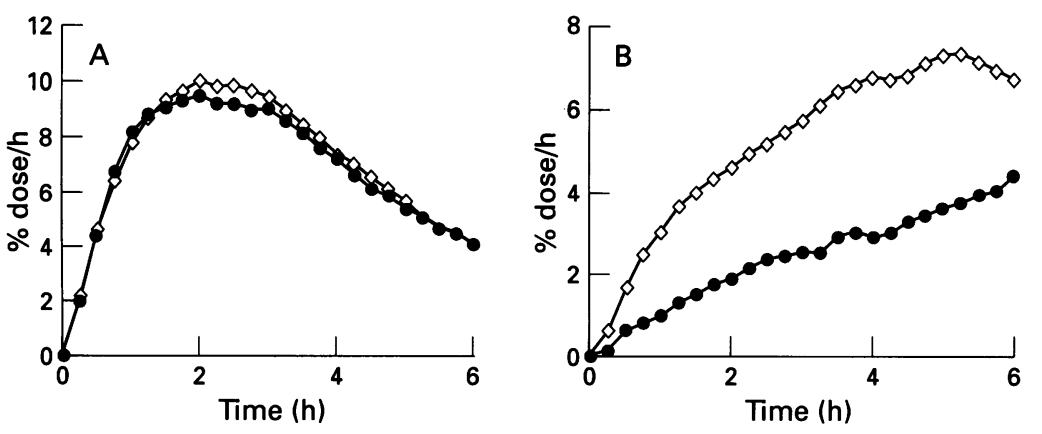

Figure 1: Mean ${ }^{13} \mathrm{CO}_{2}$ (A), ${ }^{14} \mathrm{CO}_{2}(\mathrm{~B})$, and cumulative ${ }^{14} \mathrm{CO}_{2}(C)$ excretion curves in subjects without $(\diamond)(n=34)$ pancreatic disease and with (O) $(n=14)$ pancreatic disease.

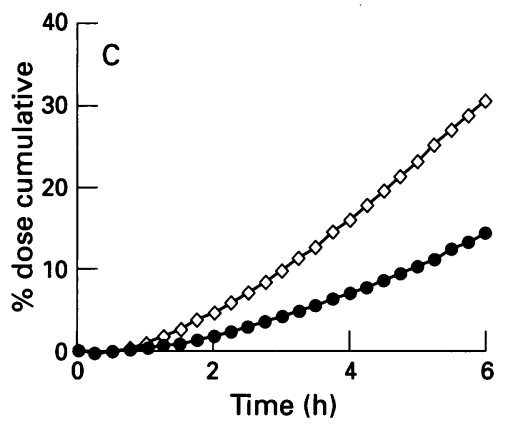

any sampling time (minimal $\mathrm{p}=0 \cdot 12$ ). On the other hand, the differences in ${ }^{14} \mathrm{CO}_{2}$ excretion rate (Fig 1B) between the two groups are highly significant (maximal $\mathrm{p}=0.05$ at $15 \mathrm{~min}$ ). The cumulative ${ }^{14} \mathrm{CO}_{2}$ excretion data are given in Fig 1C. The differences between subjects with and without pancreatic disease are statistically significant from 45 minutes to six hours (maximal $p=0.02$ ). Table I gives the results of the gastric emptying parameters derived from the ${ }^{13} \mathrm{CO}_{2}$ excretion curves and the six hour cumulative ${ }^{14} \mathrm{CO}_{2}$ excretion data of the two groups of subjects. There was no statistical difference in the GEC $(p=0 \cdot 43)$, the half emptying time $(p=0.48)$ or the lag phase $(p=0.36)$ between the subjects with and without pancreatic disease; however, the six hour cumulative ${ }^{14} \mathrm{CO}_{2}$ excretion was significantly lower in the second group $(p=0.0001)$. A correlation of 0.60 was found between the GEC and the six hour cumulative dose ${ }^{14} \mathrm{CO}_{2}$ excreted in subjects without pancreatic disease (see Fig 2). No correlation at all was detected in the group of patients with pancreatic disease $(r=0.04)$.

Because of the relation between the gastric emptying rate and the amount of ${ }^{14} \mathrm{CO}_{2}$ excreted, the group of subjects without pancreatic disease was broken down into a subgroup with normal and a subgroup with delayed gastric emptying rate, based on a previous gastric emptying study using a $250 \mathrm{kcal}$ test meal. Also the group with pancreatic disease was subdivided into a subgroup with and a subgroup without pancreatic insufficiency, based on previous measured faecal fat

TABLE I Gastric emptying results and mixed triglyceride breath test results of the subjects with and without pancreatic disease (median (interquartile range))

\begin{tabular}{lllll}
\hline & $G E C$ & $t_{1 / 2}(\min )$ & tlag (min) & $\begin{array}{l}{ }^{14} C \text {-cumulative dose } \\
6 \text { hours }\end{array}$ \\
\hline No pancreatic disease & $2 \cdot 63(2 \cdot 32-3 \cdot 02)$ & $122(91-142)$ & $64(46-91)$ & $30 \cdot 83(27 \cdot 87-34 \cdot 71)$ \\
Pancreatic disease & $2 \cdot 51(2 \cdot 34-2 \cdot 95)$ & $127(80-148)$ & $74(28-101)$ & $14 \cdot 50(5 \cdot 33-26 \cdot 68)$ \\
\hline
\end{tabular}

excretion and the actual measured six hour cumulative dose ${ }^{14} \mathrm{CO}_{2}$ excreted. ${ }^{22}$

Figure 3 gives the mean ${ }^{13} \mathrm{CO}_{2}$ and ${ }^{14} \mathrm{CO}_{2}$ excretion curves of the four subgroups examined. Compared with subjects with normal gastric emptying, there was an important delay of the ${ }^{14} \mathrm{CO}_{2}$ excretion curve in the subjects with slow gastric emptying, especially between 1.5 and 4.5 hours after administration of the ${ }^{14} \mathrm{C}$-label, which could not be discriminated from that seen in patients with pancreatic disease without insufficiency. In patients with pancreatic insufficiency, an extreme delay of ${ }^{14} \mathrm{CO}_{2}$ excretion was detected.

In Table II, the corresponding gastric emptying and mixed triglyceride breath test parameters are given. There was a significant difference in cumulative ${ }^{14} \mathrm{CO}_{2}$ excretion over six hours $(p=0.05)$ between the subjects with normal gastric emptying and the subjects with delayed gastric emptying. Both subgroups with pancreatic disease emptied in a comparable way (GEC: $p=0.44, t_{1 / 2}: p=0.22$, and tlag: $\mathrm{p}=0.36$ ) despite the large difference in six hour cumulative ${ }^{14} \mathrm{CO}_{2}$ excretion $(p=0 \cdot 0029)$. No relation was found between the GEC and the six hour cumulative ${ }^{14} \mathrm{CO}_{2}$ excretion in the subgroup with pancreatic insufficiency $(r=0.07)$. In the subgroup without pancreatic insufficiency, a similar relation between those two parameters was detected $(r=0.59)$ as in patients without pancreatic disease.

There was no difference in gastric emptying rate in the subjects with (GEC: $p=0 \cdot 13, t_{1 / 2}$ : $\mathrm{p}=0.26$, and tlag: $\mathrm{p}=0.31$ ) or without (GEC: $\mathrm{p}=0.07, \mathrm{t}_{1 / 2}: \mathrm{p}=0.08$, and tlag: $\mathrm{p}=0.13$ ) pancreatic insufficiency compared with normal healthy volunteers, but both subgroups showed a significantly lower six hour cumulative ${ }^{14} \mathrm{CO}_{2}$ excretion $(\mathrm{p}=0.0001$ and $\mathrm{p}=0.04$, respectively). As expected, the subjects with delayed gastric emptying emptied the test meal at a slower rate than the subjects with pancreatic disease but without pancreatic insufficiency (GEC: $p=0.04, t_{1 / 2}: p=0.04$ ) after a similar lag phase $(p=0.09)$, but they displayed a diminished six hour cumulative ${ }^{14} \mathrm{CO}_{2}$ excretion in the same range as patients with pancreatic disease without pancreatic insufficiency $(p=0.34)$.

Figure 2 also shows the individual values of

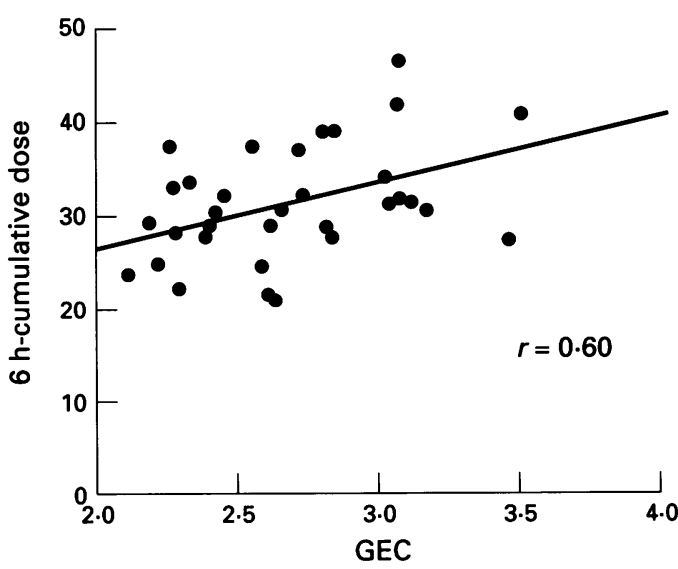

Figure 2: Relation between GEC and six hour cumulative dose ${ }^{14} \mathrm{CO}_{2}$ excreted in subjects without pancreatic disease. 

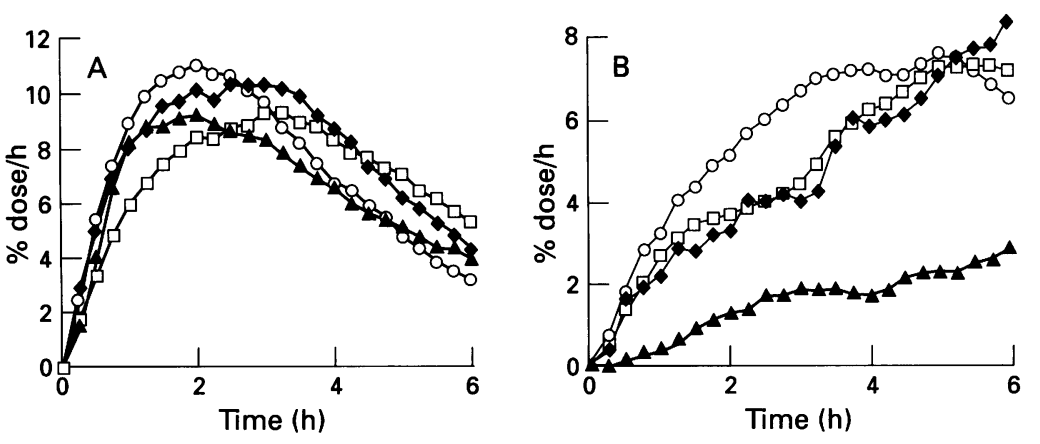

Figure 3: Mean ${ }^{13} \mathrm{CO}_{2}$ $(A),{ }^{14} \mathrm{CO}_{2}(B)$, and cumulative ${ }^{14} \mathrm{CO}_{2}(C)$ excretion curves of normal healthy volunteers $(\mathrm{O})$ $(n=21)$, subjects with delayed gastric emptying ( $\square)(n=13)$, subjects with pancreatic disease without pancreatic insufficiency $(\diamond)(n=4)$, and subjects with pancreatic disease with pancreatic insufficiency ( $\Delta)(n=10)$.

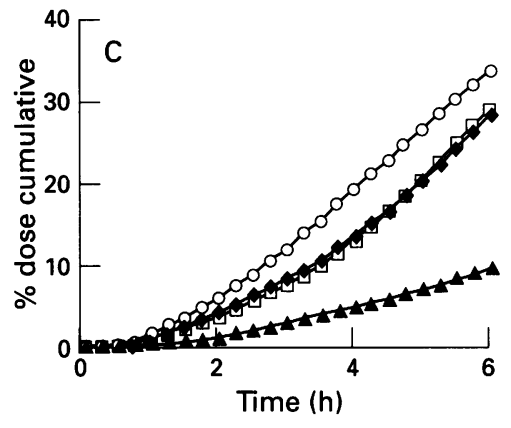

the six hour cumulative ${ }^{14} \mathrm{CO}_{2}$ excretion in the subjects without pancreatic disease. Three patients without pancreatic disease had a value below the cut off level of $22 \%$ for detection of pancreatic insufficiency. ${ }^{22}$ Given the relation between gastric emptying rate and rate of intraluminal lipolysis in subjects without pancreatic insufficiency, a correction of the ${ }^{14} \mathrm{CO}_{2}$ excretion for the gastric emptying rate was attempted in each subject using the GEC. This resulted in an increase of the ${ }^{14} \mathrm{CO}_{2}$ excretion rate in subjects with known slow gastric emptying and patients without pancreatic insufficiency; in the subjects with pancreatic insufficiency, the increase in ${ }^{14} \mathrm{CO}_{2}$ excretion was minimal (Fig 4). Consequently the cumulative ${ }^{14} \mathrm{CO}_{2}$ excretion in normal healthy volunteers, subjects with delayed gastric emptying $(p=0.25)$, and subjects with pancreatic disease without pancreatic insufficiency $(p=0.31)$ was nearly identical but better separated from the subjects with pancreatic insufficiency $(\mathrm{p}<0.0001)$ (Fig 4).

TABLE II Gastric emptying results and mixed triglyceride breath test results based on gastric emptying rate and exocrine pancreatic function (median (interquartile range)): (1) normal; (2) delayed gastric emptying; (3) pancreatic disease with pancreatic insufficiency; (4) pancreatic disease without pancreatic insufficiency

\begin{tabular}{llccc}
\hline & $G E C$ & $t_{1 / 2}(\mathrm{~min})$ & tlag $(\mathrm{min})$ & ${ }^{14}$ C-cumulative dose 6 hours \\
\hline$(1)$ & $2 \cdot 83(2 \cdot 65-3 \cdot 06)$ & $97(76-119)$ & $48(35-63)$ & $32 \cdot 74(29 \cdot 11-37 \cdot 51)$ \\
$(2)$ & $2 \cdot 28(2 \cdot 22-2 \cdot 38)$ & $154(138-199)$ & $110(75-118)$ & $28 \cdot 16(24 \cdot 94-30 \cdot 36)$ \\
$(3)$ & $2 \cdot 69(2 \cdot 27-3 \cdot 03)$ & $103(77-171)$ & $67(28-99)$ & $8 \cdot 99(5 \cdot 12-16 \cdot 15)$ \\
$(4)$ & $2 \cdot 49(2 \cdot 38-2 \cdot 76)$ & $135(113-142)$ & $79(40-103)$ & $28 \cdot 17(27 \cdot 14-29 \cdot 03)$ \\
\hline
\end{tabular}

TABLE III Gastric emptying results and mixed triglyceride breath test results based on the gastric emptying rate (median (interquartile range)): (1) normal; (2) delayed gastric emptying; (5) pancreatic disease without gastroparesis; (6) pancreatic disease with gastroparesis

\begin{tabular}{llccl}
\hline & $G E C$ & $t_{1 / 2}(\mathrm{~min})$ & tlag $(\mathrm{min})$ & ${ }^{14}$ C-cumulative dose 6 hours \\
\hline$(1)$ & $2 \cdot 83(2 \cdot 65-3 \cdot 06)$ & $97(76-119)$ & $48(35-63)$ & $32 \cdot 74(29 \cdot 11-37 \cdot 51)$ \\
$(2)$ & $2 \cdot 28(2 \cdot 22-2 \cdot 38)$ & $154(138-199)$ & $110(75-118)$ & $28 \cdot 16(24 \cdot 94-30 \cdot 36)$ \\
$(5)$ & $2 \cdot 95(2 \cdot 94-3 \cdot 19)$ & $80(77-91)$ & $28(21-44)$ & $11 \cdot 33(5 \cdot 12-26 \cdot 68)$ \\
$(6)$ & $2 \cdot 34(1 \cdot 82-2 \cdot 41)$ & $136(130-244)$ & $99(90-195)$ & $16 \cdot 15(6 \cdot 65-21 \cdot 73)$ \\
\hline
\end{tabular}

From another point of view, the group with pancreatic disease, could also be divided into two subgroups with regard to gastric emptying: seven subjects displayed a rapid gastric emptying rate (slightly faster than the normal control group without gastroparesis especially because of a significantly shorter lag phase of 28 minutes (GEC: $\mathrm{p}=0 \cdot 19, \mathrm{t}_{1 / 2}: \mathrm{p}=0 \cdot 12$, tlag: $\mathrm{p}=0.03)$ ) and seven showed a delayed gastric emptying rate comparable with subjects without pancreatic disease but with gastroparesis (GEC: $\mathrm{p}=0.42, \mathrm{t}_{1 / 2}: \mathrm{p}=0.44$, tlag: $\mathrm{p}=0.38$ ) (Table III).

\section{Discussion}

Gastric emptying is a physiological important motor function of the stomach, as the gastric emptying rate determines the rate at which nutrients are delivered to and assimilated by the small intestine. The aim of this study was to show the effect of the gastric emptying rate of a test meal on the rate of intraluminal lipolysis, using a double carbon labelled breath test. Gastric emptying was measured by the ${ }^{13} \mathrm{C}$ labelled octanoic acid breath test, which has recently been validated against the radioscintigraphic method. ${ }^{19}$ The test is able to describe the gastric emptying rate of a standard test meal in an accurate way. The rate of intraluminal lipolysis was measured by the ${ }^{14} \mathrm{C}$ labelled mixed triglyceride breath test, which earlier proved to be a reliable non-invasive test of pancreatic lipase activity in the duodenum. ${ }^{22}$ The mixed triglyceride is composed of glycerol esterified in the 1 and 3 position to stearin and in the 2 position to ${ }^{14} \mathrm{C}$-octanoic acid. Once liberated from the glycerol, ${ }^{14} \mathrm{C}$ octanoic acid is metabolised in the same way as ${ }^{13} \mathrm{C}$-octanoic acid; only emulsification and hydrolysis of the lipid makes the differences in ${ }^{13} \mathrm{CO}_{2}$ and ${ }^{14} \mathrm{CO}_{2}$ excretion rate.

In this study a test meal of $350 \mathrm{kcal}$ was used containing $19 \mathrm{~g}$ of fat. In the preparation for this study, the mixed triglyceride breath test, using $22 \%$ cumulative ${ }^{14} \mathrm{CO}_{2}$ excretion in six hours as cut off point, detects impaired rate of intraluminal lipolysis in subjects who are, because of lipase deficiency, not able to

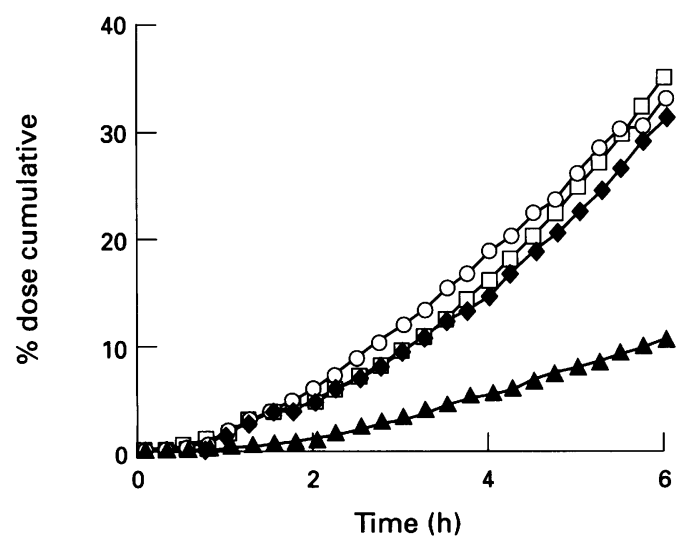

Figure 4: Mean cumulative ${ }^{14} \mathrm{CO}_{2}$ excretion curves of normal controls (O), subjects with delayed gastric emptying rate ( $\square)$, subjects with pancreatic disease without pancreatic insufficiency ( $\diamond)$, and subjects with pancreatic disease with pancreatic insufficiency $(\boldsymbol{\Delta})$, after correction for the gastric emptying rate. 
assimilate $19 \mathrm{~g}$ of fat in a normal period of time. ${ }^{22}$ By combining the ${ }^{13} \mathrm{C}$-octanoic acid breath test and the ${ }^{14} \mathrm{C}$-mixed triglyceride breath test, both gastric emptying and intraluminal lipase activity could be evaluated simultaneously in an accurate and minimally invasive way.

This study showed that in subjects without pancreatic insufficiency, gastric emptying explains about $40 \%$ of the variance of the measured intraluminal lipolysis. Because the impact of the gastric emptying rate on the six cumulative ${ }^{14} \mathrm{CO}_{2}$ excretion was statistically significant, a slow gastric emptying rate can be the rate limiting step in the rate of fat assimilation, especially when the large portion of the solid meal is emptied from the stomach (the largest difference in ${ }^{13} \mathrm{CO}_{2}$ excretion rate occurred between 1.5 and 4.5 hours after ingestion of the meal). As the rate of intraluminal hydrolysis was clearly related to the gastric emptying rate of the test meal, this finding also indirectly proves that emulsification and micellar solubilisation of the amount of administered fat in this study was not rate limiting at all in subjects with normal pancreatic function. Using the GEC, a reliable index of the overall gastric emptying rate, ${ }^{19}$ a correction of the estimated lipase activity for the emptying rate of the administered meal can be made, so that the exocrine pancreatic function can be evaluated separately - that is, based on a similar gastric emptying rate.

In patients with pancreatic insufficiency, however, gastric emptying rate of the test meal had no impact on the rate of intraluminal lipolysis. In these patients, impaired hydrolysis of the mixed triglyceride was the rate limiting step in the oxidation to ${ }^{14} \mathrm{CO}_{2}$ instead of the gastric emptying rate.

The comparison of the subjects with and without pancreatic disease showed no difference in gastric emptying rate of the test meal despite a clear distinction in fat assimilation (Fig 1). However, there seem to be two subgroups of patients with pancreatic disease with regard to gastric emptying: a subgroup with a normal gastric emptying but a very short lag phase and a subgroup with a delayed gastric emptying. No correlation was found between the gastric emptying rate and the degree of exocrine pancreatic function (lipase activity). An earlier report ${ }^{24}$ mentioned a rapid gastric emptying rate of a liquid fatty meal in seven patients with pancreatic insufficiency compared with 10 normal volunteers.

In conclusion the combined ${ }^{13} \mathrm{C}$-octanoic acid $-{ }^{14} \mathrm{C}$-mixed triglyceride breath test permits the simultaneous measurement of both the gastric emptying rate and the rate of intraluminal lipolysis. Slow gastric emptying caused a correlated decrease of the rate of intraluminal lipolysis in patients without pancreatic insufficiency. Using the gastric emptying results, the effect of slow gastric emptying on the rate of intraluminal lipolysis can be corrected for. In patients with pancreatic insufficiency, the impaired exocrine pancreatic function was the rate limiting process in the rate of fat assimilation. Additional studies, in particular, simultaneous measurement of gastric emptying may permit a more accurate evaluation of biliary, intestinal or pancreatic functions using a physiological test meal.

1 Kesteloot H, Geboes J, Pietinen P. On the within-population relationship between dietary habits and serum lipid tion relationship between dietary habits and se

2 Moreau M, Gargouri Y, Lecat D, Junien JL, Veger R. Screening of preduodenal lipases in several mammals. Biochem Biophys Acta 1988; 957: 247-52.

3 Moreau M, Laugier R, Gargouri Y, Ferrato F, Verger R. Human preduodenal lipase is entirely of gastric fundic origin. Gastroenterology 1988; 95: 1221-6.

4 Liao TH, Hamosh P, Hamosh M. Fat digestion by lingual lipase: mechanism of lipolysis in the stomach and upper small intestine. Pediatric Res 1984; 18: 402-9.

5 DeNigris SJ,. Hamosh M, Kasbekar DK, Fink CS, Lee TC, Hamosh P. Secretion of human gastric lipase from dispersed gastric glands. Biochem Biophys Acta 1985; 836: persed

6 Patton JS. Gastrointestinal lipid digestion in physiology of the gastrointestinal tract. In: Johnson LR, et al, ed. Physiology of the gastrointestinal tract. New York: Raven Press, 1981: $1123-46$.

7 Wollaeger EE. Fat, faeces and the importance of the ileum. Proc Mayo Clin 1973; 48: 833-43.

8 Rigler MW, Honkanen RE, Patton JM. Visualisation by freeze fracture, in vitro and in vivo of the products of fat digestion. $\mathcal{f}$ Lipid Res 1986; 27: 836-57.

9 Thomson ABR, Dietschy JM. Intestinal lipid absorption: major extracellular and intracellular events. In: Johnson LR, et al, ed. Physiology of the gastrointestinal tract. New LR, et al, ed. Physiology of the gastront

10 Porter HP, Saunders DR, Tytgat C, Brunser O, Rubin CE. Fat absorption in bile fistula man: a morphological and biochemical study. Gastroenterology 1971; 60: 1008-19.

11 Frederikson B, Blackberg L. Lingual lipase: an important lipase in the digestion of dietary lipids in cystic fibrosis. Pediatr Res 1980; 14: 1387-90.

12 Tanford C. The hydrophobic effect. New York: John Wiley, 1980: 1-233.

13 Beaumont W. Experiments and observations on the gastric juice and the physiology of digestion. Pittsburgh: FP Allen, 1833: 261-4.

14 Patton JS, Rigler MW, Liao TH, Hamosh P, Hamosh M. Hydrolysis of triglyceride emulsions by lingual lipase: a Hydrolysis of triglyceride emulsions by lingual lipase: a
microscopic study. Biochem Biophys Acta 1982; 712: 400-7.

15 Carey MC, Small DM, Bliss CM. Lipid digestion and absorption. Ann Rev Physiol 1983; 45: 651-77.

16 Cortot A, Phillips SF, Malagelada JR. Gastric emptying of lipids after ingestion of a solid liquid meal in human. Gastroenterology 1981; 80: 922-7.

17 Meyer JH, Mayer EA, Jehn D, Gu Y, Fink AS, Fried M. Gastric processing and emptying of fat. Gastroenterology 1986; 90: 1176-87.

18 Edelbroek M, Horowitz M, Maddox A, Bellen J. Gastric emptying and intragastric distribution of oil in the presence of a liquid or a solid meal. 7 Nucl Med 1992; 33: 1283-90.

19 Ghoos YF, Maes BD, Geypens BJ, Mys G, Hiele MI, Rutgeerts PJ, et al. Measurement of gastric emptying rate of solids by means of a carbon labelled octanoic acid of solids by means of a carbon labelled octan

20 DiMagno EP, Clain JE. Chronic pancreatitis. In: Go VLW, Gardner JD, Brooks FP, Lebenthal E, DiMagno EP, Scheele GA, eds. The exocrine pancreas: biology, pathology, and diseases. New York: Raven Press, 1986: 541-75.

21 Haycock G, Schwartz G, Wistosky D. Geometric method for measuring body surface area: a height-weight formula validated in infants, children and adults. $\mathcal{F}$ Pediatr 1978 ; 93: $62-6$.

22 Vantrappen GR, Rutgeerts PJ, Ghoos YF, Hiele MI. Mixed triglyceride breath test: a noninvasive test of pancreatic triglyceride breath test: a noninvasive test of pancreatic lipase activity.

23 SAS Institute Inc. SAS/STAT User's Guide. Release 6.03 Edition 1. Raleigh, NC: SAS Institute, 1988.

24 Long WB, Weiss JB. Rapid gastric emptying of fatty meals in pancreatic insufficiency. Gastroenterology 1974; 67: 920-5. 